\title{
A TOPOLOGY ON QUANTUM LOGICS
}

\author{
SYLVIA PULMANNOVÁ AND ZDENKA RIEČANOVÁ
}

(Communicated by Louis J. Ratliff, Jr.)

\begin{abstract}
A uniform topology $\tau_{M}$ induced by a set $M$ of finite measures on a quantum logic $L$ is studied. If $m$ is a valuation on $L$, the topology $\tau_{m}$ induced by $\{m\}$ is equivalent to the topology induced by the pseudometric $\rho(a, b)=m(a \Delta b)$. If the set $M$ of measures is large enough, the topology $\tau_{M}$ reflects in some sense the structure of $L:$ if $L$ is a continuous geometry and the measures are totally additive, $\tau_{M}$ is weaker than the order topology $\tau_{o}$ on $L$. If $L$ is atomic, $\tau_{M}$ is stronger than $\tau_{o}$. On a separable Hilbert space logic, $\tau_{M}$ coincides with the discrete topology. Some cases are found in which $\tau_{M}=\tau_{o}$.
\end{abstract}

\section{INTRODUCTION}

A finite measure $\omega$ on a Boolean algebra $B$ induces a pseudometric topology on $B$ by the function $\rho_{\omega}(a, b)=\omega(a \Delta b)$, where $\Delta$ denotes the symmetric difference. In the quantum logic approach, the function $\rho_{\omega}$ is a pseudometric iff $\omega$ is subadditive, or equivalently, iff $\omega$ is a valuation [5]. However, there are many important examples of quantum logics having no subadditive finite measure.

In [5], a uniform topology $\tau_{\omega}$ induced by a finite measure $\omega$ on a quantum logic (=orthomodular $\sigma$-lattice) $L$ has been introduced. It has been shown that provided that $\omega$ is a valuation (e.g. if $L$ is a Boolean algebra), the topology $\tau_{\omega}$ coincides with the topology induced by the pseudometric $\rho_{\omega}$. In the present paper, we study properties of a uniform topology $\tau_{M}$ induced by a set $M$ of finite measures on a logic $L$. We compare the $\tau_{M}$ topology with the order topology $\tau_{o}$ on $L$.

\section{BASIC FACTS AND DEFINITIONS}

By a (quantum) logic we mean an orthomodular $\sigma$-lattice $L$ (see [7, p. 42] for the definition). For $a \in L$ we denote by $a^{\perp}$ the orthocomplement of $a$.

Received by the editors July 8, 1988 and, in revised form, November 8, 1988.

1980 Mathematics Subject Classification (1985 Revision). Primary 06B30, 06C15, 81 B10, 03G12.

Key words and phrases. Orthomodular lattice, quantum logic, uniform topology generated by measures. 
The symmetric difference of the elements $a, b \in L$ is the element $a \Delta b=$ $\left.(a \wedge(a \wedge b))^{\perp}\right) \vee\left(b \wedge(a \wedge b)^{\perp}\right)$. Orthomodularity of $L$ is equivalent to $a \vee b=$ $(a \wedge b) \vee(a \Delta b)$, and therefore $a \Delta b=(a \vee b) \wedge(a \wedge b)^{\perp}$. Two elements $a, b$ of $L$ are orthogonal if $a \leq b^{\perp}$ (we write $a \perp b$ ). A (finite) measure on $L$ is a map $m: L \rightarrow \mathbf{R}^{+}$such that $m(0)=0$ and $m\left(\vee_{i \in \mathbf{N}} a_{i}\right)=\sum_{i \in \mathbf{N}} m\left(a_{i}\right)$ for any sequence $\left(a_{i}\right)_{i \in \mathbf{N}}$ of mutually orthogonal elements of $L$. A measure $m$ on $L$ is a valuation if $m(a \vee b)+m(a \wedge b)=m(a)+m(b)$ for all $a, b \in L$. It is easy to prove that $m$ is a valuation iff it is subadditive, that is $m(a \vee b) \leq m(a)+m(b)$. (For more details on logics see [1], [2], [3], [6], [7].)

Let $M$ be a set of finite measures on $L$. We say that $M$ is

-separating if $a \neq 0 \Rightarrow \exists m \in M: m(a) \neq 0$;

-unital if $a \neq 1 \Rightarrow \exists m \in M: m(a)=0$;

一ordering if $a \ddagger b \Rightarrow \exists m \in M: m(a)>m(b)$.

Clearly, if $M$ is ordering or unital, it is separating. A measure $\omega$ on $L$ is faithful if $\{\omega\}$ is separating.

Let $\omega$ be a finite measure on $L$. For every $x \in L$ we define the mappings $\rho_{x \vee}: L x L \rightarrow \mathbf{R}^{+}, \rho_{x \wedge}: L x L \rightarrow \mathbf{R}^{+}$by

$$
\begin{aligned}
& \rho_{x \vee}(a, b)=|\omega(a \vee x)-\omega(b \vee x)|, \\
& \rho_{x \wedge}(a, b)=|\omega(a \wedge x)-\omega(b \wedge x)| .
\end{aligned}
$$

It is easy to verify that $D=\left\{\rho_{x \vee} \mid x \in L\right\} \cup\left\{\rho_{x \wedge} \mid x \in L\right\}$ forms a collection of pseudometrics on $L$. Thus the collection $D$ induces some uniformity on $L$ which is compatible with some topology on $L$. Denote this topology by $\tau_{\omega}$. Then for a net $\left(a_{\alpha}\right)_{\alpha \in A}$ of points of the topological space $\left(L, \tau_{\omega}\right)$ and $a \in L$ it holds $a_{\alpha} \stackrel{\tau_{\omega}}{\rightarrow} a$ iff $\omega\left(a_{\alpha} \vee x\right) \rightarrow \omega(a \vee x)$ and $\omega\left(a_{\alpha} \wedge x\right) \rightarrow \omega(a \wedge x)$ for every $x \in L$. In other words, $\tau_{\omega}$ is the weakest topology in which all the functionals $f_{x \vee}(a)=\omega(a \vee x), f_{x \wedge}(a)=\omega(a \wedge x)$ are continuous for every $x \in L$. In [5], following properties of the topology $\tau_{\omega}$ have been proved.

Theorem 1. Let $L$ be a logic and let $\omega: L \rightarrow \mathbf{R}^{+}$be a measure. Let $\tau_{\omega}$ be the uniform topology on $L$ induced by $\omega$. Then

(i) $a_{\alpha} \stackrel{\tau_{\omega}}{\rightarrow} a \Rightarrow a_{\alpha}^{\perp} \stackrel{\tau_{\omega}}{\rightarrow} a^{\perp}$.

(ii) $a_{\alpha} \stackrel{\tau_{\omega}}{\rightarrow} a \Rightarrow \omega\left(a_{\alpha} \Delta a\right) \rightarrow 0$.

(iii) $a_{\alpha} \stackrel{\tau_{\omega}}{\rightarrow} a, b_{\alpha} \stackrel{\tau_{\omega}}{\rightarrow} b, a_{\alpha} \perp b_{\alpha}, a \perp b \Rightarrow \omega\left(a_{\alpha} \vee b_{\alpha}\right) \rightarrow \omega(a \vee b)$.

(iv) $a_{\alpha} \stackrel{\tau_{\omega}}{\rightarrow} a \Rightarrow m\left(a_{\alpha}\right) \rightarrow m(a)$ for every signed measure $m: L \rightarrow\langle-\infty, \infty\rangle$ such that $m \ll_{\varepsilon} \omega$.

(v) $\forall \alpha \in A: b_{\alpha} \leq a_{\alpha} \leq c_{\alpha}$ and $b_{\alpha} \stackrel{\tau_{\omega}}{\longrightarrow} a, c_{\alpha} \stackrel{\tau_{\omega}}{\longrightarrow} a \Rightarrow a_{\alpha} \stackrel{\tau_{\omega}}{\longrightarrow} a$.

(vi) If $\omega$ is a valuation (or equivalently, if $\omega$ is subadditive) then $a_{\alpha} \stackrel{\tau_{\omega}}{\longrightarrow} a$ iff $\omega\left(a_{\alpha} \Delta a\right) \rightarrow 0$.

(vii) If $\omega$ is faithful, $\tau_{\omega}$ is Hausdorff.

Proof. (i)-(v) are left to the reader.

(vi). Let $\omega$ be a valuation. If $a_{\alpha} \stackrel{\tau_{\omega}}{\rightarrow} a$, then $\omega\left(a_{\alpha} \vee a\right) \rightarrow \omega(a), \omega\left(a_{\alpha} \wedge a\right) \rightarrow$ $\omega(a)$ by definition, and hence $\omega\left(a_{\alpha} \Delta a\right) \rightarrow 0$. Now let $\omega\left(a_{\alpha} \Delta a\right) \rightarrow 0$. By 
[2, X.Th.1], $\omega\left(\left(x \vee a_{\alpha}\right) \Delta(x \vee a)\right)+\omega\left(\left(x \wedge a_{\alpha}\right) \Delta(x \wedge a)\right) \leq \omega\left(a_{\alpha} \Delta a\right)$ for any $x \in L$, which implies $\omega\left(\left(x \vee a_{\alpha}\right) \Delta(x \vee a)\right) \rightarrow 0, \omega\left(\left(x \wedge a_{\alpha}\right) \Delta(x \wedge a)\right) \rightarrow 0$. It is easy to see that $\omega\left(a_{\alpha} \Delta a\right) \rightarrow 0 \Rightarrow \omega\left(a_{\alpha}\right) \rightarrow \omega(a)$. Indeed, $\omega\left(a_{\alpha} \Delta a\right)=$ $\left[\omega\left(a_{\alpha} \vee a\right)-\omega(a)\right]+\left[\omega(a)-\omega\left(a_{\alpha} \wedge a\right)\right] \rightarrow 0$. Therefore $\omega\left(a_{\alpha} \vee a\right) \rightarrow \omega(a)$, $\omega\left(a_{\alpha} \wedge a\right) \rightarrow \omega(a)$ and $\omega\left(a_{\alpha} \vee a\right) \geq \omega\left(a_{\alpha}\right) \geq \omega\left(a_{\alpha} \wedge a\right)$ implies $\omega\left(a_{\alpha}\right) \rightarrow \omega(a)$. (vii). Let $\omega$ be faithful and suppose that $a_{\alpha} \stackrel{\tau_{\omega}}{\rightarrow} a$ and $a_{\alpha} \stackrel{\tau_{\omega}}{\rightarrow} b$. Then $\omega\left(a_{\alpha} \wedge a\right) \rightarrow \omega(a)$ and $\omega\left(a_{\alpha} \wedge a\right) \rightarrow \omega(a \wedge b)$ imply that $\omega(a)=\omega(a \wedge b)$. By orthomodularity, $a=a \wedge b \vee a \wedge(a \wedge b)^{\perp}$. We get $\omega\left(a \wedge(a \wedge b)^{\perp}\right)=0$, and since $\omega$ is faithful, this entails $a=a \wedge b$. Similarly we obtain that $b=a \wedge b$, and hence $a=b$. This concludes the proof.

We note that in [4] a topology $\tau_{a s}$ induced by a finite measure $\omega$ on a logic $L$ such that $a_{\alpha} \stackrel{\tau_{a s}}{\rightarrow} a \Leftrightarrow \forall \ll_{\varepsilon} \omega: m\left(a_{\alpha}\right) \rightarrow m(a)$ has been studied. From (iv) it follows that $\tau_{\omega} \supset \tau_{a s}$.

Remark 1 . For any $x \in L$, denote by $\varphi_{x}$ the Sasaki projection $\varphi_{x}(a)=$ $\left(a \vee x^{\perp}\right) \wedge x(a \in L)$. It is easy to prove that for any finite measure $\omega$, the map $\omega \circ \varphi_{x}: L \rightarrow \mathbf{R}^{+}$is continuous in $\tau_{\omega}$. Also, $a_{\alpha} \stackrel{\tau_{\omega}}{\rightarrow} a$ iff $\omega \circ \varphi_{x}\left(a_{\alpha}\right) \rightarrow \omega \circ \varphi_{x}(a)$ and $\omega \circ \varphi_{x}\left(a_{\alpha}^{\perp}\right) \rightarrow \omega \circ \varphi_{x}\left(a^{\perp}\right)$ for all $x \in L$.

\section{RELATIONS BETWEEN $\tau_{M}$-TOPOLOGY AND THE ORDER TOPOLOGY}

Let $M$ be a set of finite measures on a logic $L$. We define a $\tau_{M}$-topology as follows: $a_{\alpha} \stackrel{\tau_{M}}{\rightarrow} a$ if $a_{\alpha} \stackrel{\tau_{m}}{\rightarrow} a$ for any $m \in M$. Let $L$ be a complete orthomodular lattice (=complete quantum logic). The net $\left(a_{\alpha}\right)_{\alpha}(o)$-converges to $a$ (written $a_{\alpha} \stackrel{(o)}{\rightarrow} a$ ) if $\lim \sup a_{\alpha}=\liminf a_{\alpha}=a$, where $\lim \sup a_{\alpha}=\wedge_{\beta} \vee_{\alpha \geq \beta} a_{\alpha}$, $\liminf a_{\alpha}=\vee_{\beta} \wedge_{\alpha \geq \beta} a_{\alpha}$. The order topology $\tau_{o}$ on $L$ is the strongest topology such that $(o)$-convergence of a net implies the topological convergence. If we consider instead of nets only sequences, we obtain (os)-convergence and $\tau_{o s}$-topology (=sequential order topology). In general, $\tau_{o s} \supset \tau_{o}$. The equality $\tau_{o s}=\tau_{o}$ in $L$ holds iff $L$ is separable (i.e. if any set of pairwise orthogonal nonzero elements of $L$ is at most countable) (see [2], [6]). An element $a$ of $L$ is an atom if $a$ covers 0 (i.e. $0 \leq b \leq a$ implies $b=a$ or $b=0$ ). A logic $L$ is atomic if every element of $L$ contains an atom, and $L$ is atomistic if every element in $L$ is the supremum of all atoms lying under it. It is easy to prove that any atomic orthomodular lattice is atomistic. A logic $L$ is $(o)$-continuous if $a_{\alpha} \nearrow a$ implies $a_{\alpha} \wedge b \nearrow a \wedge b$ for every $b \in L$ (where $a_{\alpha} \nearrow a$ means that the net $\left(a_{\alpha}\right)_{\alpha}$ is nondecreasing and $\left.\vee a_{\alpha}=a\right)$. By duality we obtain that $a_{\alpha} \searrow a$ implies $a_{\alpha} \vee b \searrow a \vee b$ for every $b \in L$. We note that if $L$ is complete and modular, it is a continuous geometry (a well-known result of Kaplansky), and therefore it is $(o)$-continuous.

The definition of ( 0$)$-convergence can be generalized to logics not necessarily complete. In such a case, (0)-convergence is defined as follows: $a_{\alpha} \stackrel{(0)}{\rightarrow} a$ if there are nets $\left(b_{\alpha}\right)_{\alpha},\left(c_{\alpha}\right)_{\alpha}$ such that $b_{\alpha} \leq a_{\alpha} \leq c_{\alpha}$ for every $\alpha$ and $b_{\alpha} \nearrow a$, $c_{\alpha} \nearrow a$ (see [2], [6]). 
A measure $m$ on $L$ is completely additive if $m\left(\vee_{\alpha} a_{\alpha}\right)=\sum m\left(a_{\alpha}\right)$ for any set $\left(a_{\alpha}\right)_{\alpha}$ of pairwise orthogonal elements in $L$. Complete additivity is equivalent to $m\left(a_{\alpha}\right) \rightarrow m(a)$, provided that $a_{\alpha} \nearrow a$.

Theorem 2. Let $L$ be a logic and let $M$ be a separating set of finite measures on $L$. If $\tau_{0} \supset \tau_{M}$, then all the measures in $M$ are completely additive and $L$ is (0)-continuous.

Proof. If $\tau_{0} \supset \tau_{M}$ then $x_{\alpha} \nearrow x$ implies $m\left(x_{\alpha}\right) \rightarrow m(x)$ for all $m \in M$, hence all the measures in $M$ are completely additive.

Suppose that $L$ is not (0)-continuous. Then there is a net $\left(x_{\alpha}\right)_{\alpha}$ and $y$ in $L$ such that $x_{\alpha} \nearrow x$ and $x_{\alpha} \wedge y \not \gamma \wedge y$. Suppose that $x_{\alpha} \stackrel{\tau_{M}}{\rightarrow} x$. Then $m\left(x_{\alpha} \wedge y\right) \rightarrow m(x \wedge y)$ for all $m \in M$. Evidently, $x_{\alpha} \wedge y \leq x \wedge y$ for all $\alpha$. Let $d \in L$ be such that $x_{\alpha} \wedge y \leq d$ for all $\alpha$. Then we have $m(x \wedge y)=$ $\lim _{\alpha} m\left(x_{\alpha} \wedge y\right) \leq m(d \wedge x \wedge y)$ for all $m \in M$. As $M$ is separating, we get $x \wedge y=d \wedge x \wedge y$, i.e. $\bigvee x_{\alpha} \wedge y=x \wedge y$, in contradiction with the supposition. Hence $x_{\alpha} \stackrel{(\sigma)}{\rightarrow} x$, but $x_{\alpha} \stackrel{\tau_{M}}{\rightarrow} x$.

Corollary 1. If $M$ is a separating set of finite measures on a complete logic $L$, then $\tau_{0} \supset \tau_{M}$ iff all the measures in $M$ are completely additive and the logic $L$ is (0)-continuous.

Theorem 3. Let $L$ be an atomic logic. Let $M$ be a separating set of finite measures on $L$. Then $\tau_{M} \supset \tau_{o}$.

Proof. Observe that any $a \in L$ is the supremum of maximal set of orthogonal atoms lying under $a$. To show this, let $\left(x_{\nu}\right)_{\nu}$ be a maximal set of pairwise orthogonal atoms such that $x_{\nu} \leq a$. Let $b \in L$ be such that $x_{\nu} \leq b$ for all $\nu$. Then $x_{\nu} \leq a \wedge b$. If $b \wedge a \neq a$, there is an atom $y \leq(a \wedge b)^{\perp} \wedge a$. Then $y \leq a, y \leq a^{\perp} \vee b^{\perp} \leq x_{\nu}^{\perp}$ for all $\nu$, which contradicts the maximality of $\left(x_{\nu}\right)_{\nu}$. Now let $G$ be a $\tau_{o}$-closed subset of $L$. Let $\left(a_{\alpha}\right)_{\alpha \in A}$ be a net such that $a_{\alpha} \in G$ for all $\alpha \in A$ and $a_{\alpha} \stackrel{\tau_{M}}{\rightarrow} a$. Let $\left(x_{\nu}\right)_{\nu},\left(y_{\mu}\right)_{\mu}$ be sets of pairwise orthogonal atoms such that $\bigvee x_{\nu}=a, \bigvee y_{\mu}=a^{\perp}$. For every $m \in M$, there is an at most countable subset $\left(x_{n}^{m}\right)$ of $\left(x_{\nu}\right)$ such that $m\left(x_{n}^{m}\right) \neq 0$ and $m\left(x_{\nu}\right)=0$ if $x_{\nu} \neq x_{n}^{m}, n \in \mathbf{N}$. Since $m\left(x_{\nu} \wedge a_{\alpha}\right) \rightarrow m\left(x_{\nu} \wedge a\right)$ for all $\nu$, we obtain that there is a $\beta_{1}(n, m) \in A$ such that for all $\alpha \geq \beta_{1}(n, m)$ we have $x_{n}^{m} \leq a_{\alpha}$. We may suppose that $\beta_{1}(1, m) \leq \beta_{1}(2, m) \leq \cdots$. Similarly, there is a $\beta_{2}(n, m)$ such that $y_{n}^{m} \leq a_{\alpha}^{\perp}$ for $\alpha \geq \beta_{2}(n, m)$, and we may suppose that $\beta_{2}(1, m) \leq \beta_{2}(2, m) \leq \cdots$. Let $\mathscr{F}$ be the set of all finite subsets of $M$. For a given $F \in \mathscr{F}$ and a given $n \in \mathbf{N}$, we choose $\gamma(n, F) \in A$ such that $\gamma(n, F) \geq \beta_{i}(n, m)$ for all $m \in F$ and $i=1,2$. Then for any $\alpha \geq \gamma(n, F)$ we have $\bigvee_{m \in F} \bigvee_{i \leq n} x_{i}^{m} \leq a_{\alpha} \leq\left(\bigvee_{m \in F} \bigvee_{i \leq n} y_{i}^{m}\right)^{\perp}$. Let $B=\{(n, F) \mid n \in \mathbf{N}$, $F \in \mathscr{F}\}$. Define a partial ordering on $B$ by putting $\left(n_{1}, F_{1}\right) \leq\left(n_{2}, F_{2}\right)$ iff $n_{1} \leq n_{2}$ and $F_{1} \subset F_{2}$. Then $B$ with this ordering is a directed set. The net $\left(\bigvee_{m \in F} \bigvee_{i \leq n} x_{i}^{m}\right)_{(n, F) \in B}$ is nondecreasing and $\bigvee_{m \in F} \bigvee_{i \leq n} x_{i}^{m} \nearrow a$. Indeed, 
suppose that $\bigvee_{m \in F} \bigvee_{i \leq n} x_{i}^{m} \leq b \wedge a$ for all $(n, F) \in B$. If $b \wedge a<a$, there is an atom $z \leq a, z \leq(b \wedge a)^{\perp} \leq\left(x_{i}^{m}\right)^{\perp}$ for all $i \in \mathbf{N}$ and all $m \in M$. But this implies that $z \perp x_{\nu}$ for all $\nu$ (since $M$ is separating, to every $x_{\nu}$ there is $m \in M$ such that $\left.m\left(x_{\nu}\right) \neq 0\right)$, which contradicts the maximality of $\left(x_{\nu}\right)_{\nu}$. In a similar way we obtain that the net $\left(\bigvee_{m \in F} \bigvee_{i \leq n} y_{i}^{m}\right)_{(n, F) \in B}$ is nondecreasing and $\bigvee_{m \in F} \bigvee_{i \leq n} y_{i}^{m} \nearrow a^{\perp}$. Thus $\left(a_{(n, F)}\right)_{(n, F) \in B}$, where $a_{(n, F)}=a_{\gamma(n, F)}$ for all $(n, F) \in B$, is a net of points of $G$ such that $a_{(n, F)} \stackrel{(o)}{\rightarrow} a$. Hence $a \in G$, i.e., $G$ is also $\tau_{M}$-closed. This proves that $\tau_{M} \supset \tau_{o}$.

We say that $L$ satisfies a finite chain condition $(f c c)$ if every chain in $L$ has a finite length. If $L$ satisfies $f c c$, it is atomic, $(o)$-continuous and separable. This implies the following.

Corollary 2. If $L$ satisfies $f c c$ and $M$ is a separating set of finite measures on $L$, then $\tau_{o s}=\tau_{o}=\tau_{M}$ and all these topologies are discrete.

Corollary 3. Let $L$ be an atomic logic and $\omega$ be a faithful finite measure on $L$. Then $\tau_{\omega} \supset \tau_{o}=\tau_{o s}$. If $L$ is also (o)-continuous, then $\tau_{\omega}=\tau_{o}=\tau_{o s}$.

Proof. Let $\left(a_{\nu}\right)_{\nu}$ be a maximal set of pairwise orthogonal elements in $L$. There is an at most countable subset $\left(a_{n}\right)_{n \in \mathbf{N}}$ of $\left(a_{\nu}\right)_{\nu}$ such that $\omega\left(a_{\nu}\right)=0$ if $a_{\nu} \neq$ $a_{n}, n \in \mathbf{N}$. Since $\omega$ is faithful, $a_{\nu}=0$ for $a_{\nu} \neq a_{n}, n \in \mathbf{N}$. Hence $L$ is separable so that $\tau_{o s}=\tau_{o}$. Theorem 3 implies that $\tau_{\omega} \supset \tau_{o}$. The rest of the proof is straightforward.

Theorem 4. Let $L$ be an (o)-continuous logic and let $M$ be a finite ordering set of finite measures on $L$. Then $\tau_{M}=\tau_{o s}$.

To prove this assertion, we need two lemmas.

Lemma 1. Let $\left(x_{\alpha}\right)_{\alpha \in A}$ be a net of nonnegative numbers such that $x_{\alpha} \rightarrow 0$. Then two cases can occur:

(i) $\exists \alpha_{0} \forall \alpha \geq \alpha_{0}: x_{\alpha}=0$;

(ii) $\exists \alpha_{1} \leq \alpha_{2} \leq \ldots$ such that $x_{\alpha_{1}}>x_{\alpha_{2}}>\ldots$ and $x_{\alpha_{n}} \searrow 0$.

Lemma 2. Let $M$ be a finite ordering set of finite measures on a logic L. Let $\left(a_{\alpha}\right)_{\alpha \in A}$ be a net of $L$ such that $m\left(a_{\alpha}\right) \rightarrow 0$ for all $m \in M$. Two cases can occur:

(i) $\exists \alpha_{0} \forall \alpha \geq \alpha_{0}: a_{\alpha}=0$;

(ii) $\exists \alpha_{1} \leq \alpha_{2} \leq \ldots$ such that $a_{\alpha_{n}} \stackrel{(o)}{\rightarrow} 0$.

Proof. Let $M=\left\{m_{1}, m_{2}, \ldots, m_{n}\right\}$. Define $\varphi=m_{1}+m_{2}+\cdots+m_{n}$. Then $\varphi\left(a_{\alpha}\right) \rightarrow 0$ and in view of Lemma 1 :

-Either, there is an index $\alpha_{0} \in A$ such that for every $\alpha \geq \alpha_{0}$ it holds $\varphi\left(a_{\alpha}\right)=$ 0 . Thus $m_{k}\left(a_{\alpha}\right)=0$ for every $m_{k} \in M$ and hence $a_{\alpha}=0$.

-Or, there is a subsequence $\left(a_{\alpha_{n}}\right)_{n \in \mathbf{N}}$ such that $\varphi\left(a_{\alpha_{n}}\right) \rightarrow 0$ and hence $m_{k}\left(a_{\alpha_{n}}\right)$ $\rightarrow 0$ for all $m_{k} \in M$. By induction with respect to the number of elements of 
$M$ using Lemma 1 we can choose a subsequence $\left(a_{\alpha_{n, j}}\right)_{j \in \mathbf{N}}$ such that for every $m_{k} \in M$ it holds that $m_{k}\left(a_{\alpha_{n, 1}}\right) \geq m_{k}\left(a_{\alpha_{n, 2}}\right) \geq \cdots \searrow 0$. Since $M$ is ordering we obtain $a_{\alpha_{n, 1}} \geq a_{\alpha_{n, 2}} \geq \ldots$. Suppose that there is an element $b \in L, b \neq 0$, such that $b \leq a_{\alpha_{n, j}}, j \in \mathbf{N}$. Then there is $m_{k} \in M$ such that $m_{k}(b)>0$ which contradicts $m_{k}\left(a_{\alpha_{n, j}}\right) \rightarrow 0$. Hence $\bigwedge_{j \in \mathbf{N}} a_{\alpha_{n, j}}=0$ and thus $a_{\alpha_{n, j}} \stackrel{(o)}{\rightarrow} 0$.

Proof of Theorem 4. Let $G$ be a $\tau_{o s}$-closed subset of $L$. Let $a_{\alpha} \in G$ for all $\alpha$ and let $a_{\alpha} \stackrel{\tau_{M}}{\rightarrow} a$. Then by Theorem 1(ii) $m\left(a_{\alpha} \Delta a\right) \rightarrow 0$ for all $m \in M$. In view of Lemma 2 there exists a subsequence $\left(a_{\alpha_{k}}\right)_{k \in \mathbf{N}}$ such that $a_{\alpha_{k}} \Delta a \stackrel{(o)}{\rightarrow} 0$ and, since $L$ is $(o)$-continuous, we obtain $a_{\alpha_{k}} \stackrel{(o)}{\rightarrow} a$ (see [6]). This proves that $\tau_{M} \supset \tau_{o s}$. From the $(o)$-continuity of $L$ we obtain that $\tau_{M}=\tau_{o s}$.

\section{4. $\tau_{M}$-TOPOLOGY ON A HILBERT SPACE LOGIC}

Let $H$ be a Hilbert space. The set $L(H)$ of all closed subspaces of $H$ forms a complete, atomistic logic which is called a Hilbert space logic. A Hilbert space logic is separable iff the Hilbert space $H$ is separable, and it satisfies $f c c$ iff $H$ is finite dimensional. Theorem 3 and Corollary 2 imply the following.

Theorem 5. Let $L(H)$ be a Hilbert space logic and let $M$ be a separating set of finite measures on $L(H)$. Then $\tau_{M} \supset \tau_{o}$, and $\tau_{M}=\tau_{o}$ iff $H$ is finite dimensional.

Theorem 6. Let $L(H)$ be a separable Hilbert space logic and let $M$ be a separating set of finite measures on $L(H)$. Then $\tau_{M}$ coincides with the discrete topology on $L(H)$.

Proof. As in the proof of Theorem 3, if $a_{\alpha} \stackrel{\tau_{M}}{\rightarrow} a$, then for every atom $x$, $x \leq a$ implies $x \leq \bigwedge_{\alpha \geq \beta(x)} a_{\alpha}$ for some $\beta(x)$. Put $b_{\beta}=\bigwedge_{\alpha \geq \beta} a_{\alpha}$. Then $\left(b_{\beta}\right)_{\beta}$ is nondecreasing, and since $L(H)$ is separable, there is a sequence $\left(b_{n}\right)_{n} \subset$ $\left(b_{\beta}\right)$ such that $\bigvee b_{n}=\bigvee b_{\beta}$ (see e.g. [6]). Suppose that $\bigvee_{n \in \mathbf{N}} b_{n} \neq b_{k}$ for all $k \in \mathbf{N}$. Choose a sequence of orthogonal vectors $u_{n} \in b_{n}-b_{n-1}, n \in$ $\mathbf{N}$. Put $v=\sum_{n=1}^{\infty} c_{n} u_{n}, 0 \neq c_{n} \in C$. Denote by [ $v$ ] the one-dimensional subspace generated by $v$. Then $[v] \not b_{n}, n \in \mathbf{N}$, but $[v] \leq \bigvee_{n \in \mathbf{N}} b_{n}=$ $a$, a contradiction. Therefore, the sequence $\left(b_{n}\right)_{n}$, and hence the net $\left(b_{\beta}\right)_{\beta}$, has a maximal element, say $b_{\beta_{0}}=a$. Therefore, $\Lambda_{\alpha \geq \beta} a_{\alpha}=\Lambda_{\alpha \geq \beta_{0}} a_{\alpha}$ for all $\beta \geq \beta_{0}$. In the same manner, using the fact that $y \leq a^{\perp}$ (y an atom) implies $y \leq \bigwedge_{\alpha \geq \beta(y)} a_{\alpha}^{\perp}$, we show that $c_{\beta}=\bigvee_{\alpha \geq \beta} a_{\alpha}=\bigvee_{\alpha \geq \beta_{1}} a_{\alpha}=a$ for all $\beta \geq \beta_{1}$. We have found two stationary nets $\left(b_{\alpha}\right),\left(c_{\alpha}\right)$ such that $b_{\alpha} \leq a_{\alpha} \leq c_{\alpha}$ for all $\alpha \geq \gamma$, where $\gamma \geq \beta_{0}, \beta_{1}$. Therefore $\left(a_{\alpha}\right)_{\alpha}$ is a stationary net.

\section{REFERENCES}

1. L. Beran, Orthomodular lattices, Academia-Reidel P.C., Dordrecht, Holland 1984.

2. G. Birkhoff, Lattice theory (Russian translation: Teorija rešetok), Moscow, "NAUKA”, 1984. 
3. G. Kalmbach, Orthomodular lattices, Academic Press, London 1983.

4. V. Palko, Topologies on quantum logics induced by measures, Math. Slovaca, to appear.

5. Z. Riečanová, Topology in a quantum logic induced by a measure, Proceedings of the Conf. "Topology and Measure V", Wissenschaftliche Beitrage der EMA-Univesität Greifswald DDR, Greifswald 1988.

6. T. A. Sarymsakov, S. A. Ajupov, Z. Chadžijev, and V. J. Čilin, Uporiadocennije algebry, Taškent, "FAN" 1983.

7. V. S. Varadarajan, Geometry of quantum theory, Springer-Verlag, New York 1985.

Mathematics Institute, Slovak Academy of Sciences, 81473 Bratislava, Czechoslovakia

Technical University of Bratislava-El. Eng., Department of Mathematics, 81219 Bratislava, Czechoslovakia 